\title{
ON TAUBERIAN CONDITIONS OF TYPE $\circ$
}

\author{
BY WERNER MEYER-KÖNIG AND HUBERT TIETZ
}

Communicated by Maurice Heins, June 6, 1967

The series $\sum a_{n}$ ( $\sum$ means $\sum_{n=0}^{\infty}$ ) is said to be summable to the sum $s$ by Abel's method of summability, if $\sum a_{n} x^{n}=f(x)$ is convergent for $0<x<1$ and if $f(x) \rightarrow s$ as $x \rightarrow 1^{-}(x$ real $)$. A classical theorem of A. Tauber [2] states that if $\sum a_{n}$ is summable to the sum $s$ by Abel's method and if

$$
a_{n}=o(1 / n) \text { as } n \rightarrow \infty
$$

then $\sum a_{n}=s$. In today's language we put this in the following way: (1) is a Tauberian condition for Abel's method (cf., e.g., Hardy [1, pp. 149-152]). Again according to Tauber [2] the weaker condition

$$
\delta_{n}=o(1) \quad \text { with } \quad \delta_{n}=(n+1)^{-1} \sum_{k=0}^{n} k a_{k}
$$

is also a Tauberian condition for Abel's method.

We shall show that Tauber's passage from (1) to (2) is possible for a very general class of summability methods. Formula (3) which yields this passage was already used by Tauber $[2$, p. 276, (6)]; here we exploit it more fully.

The summability method $V$ is said to be regular if $\sum a_{n}=s$ implies $V-\sum a_{n}=s . V$ is called additive if

$$
V-\sum a_{n}=s, \quad V-\sum b_{n}=t \text { implies } V-\sum\left(a_{n}+b_{n}\right)=s+t .
$$

THEOREM. If (1) is a Tauberian condition for the regular and additive method $V$ then also (2) is a Tauberian condition for $V$.

Proof. We assume that (1) is a Tauberian condition for $V$ and that we have under consideration a given series $\sum a_{n}$ which is summable $V$ to the sum $s$ and for which (2) is fulfilled. We have to show that $\sum a_{n}=s$. Putting $b_{0}=a_{0}$ and $b_{n}=\delta_{n} / n(n=1,2, \cdots)$ the equation

$$
a_{0}+\cdots+a_{n}=\left(b_{0}+\cdots+b_{n}\right)+\delta_{n} \quad(n=0,1, \cdots)
$$

is easily proved by induction. Together with $V-\sum a_{n}=s$ and $V$ - $\lim (-\delta n)=0$, (3) gives $V-\sum b_{n}=s$. Since $b_{n}=o(1 / n)$ we conclude that $\sum b_{n}=s$ and further, again from (3), that $\sum a_{n}=s$. 
If, a sequence $\lambda$ being given $\left(\lambda_{n-1}<\lambda_{n} \rightarrow \infty\right.$ as $\left.n \rightarrow \infty\right)$, (1) is replaced by

$$
a_{n}=o\left(1 / \lambda_{n}\right) \text { as } n \rightarrow \infty
$$

and (2) by

$$
(n+1)^{-1}\left(\lambda_{0} a_{0}+\cdots+\lambda_{n} a_{n}\right)=o(1),
$$

the theorem is still true provided that

$$
n / \lambda_{n}=O(1) \text { and } n\left(\lambda_{n+1}-\lambda_{n}\right) / \lambda_{n+1}=O(1) .
$$

Herewith the cases

$$
\lambda_{n}=n \log n, \quad \lambda_{n}=n \log n \log \log n, \cdots
$$

are covered. The theorem fails to remain true if $n / \lambda_{n} \rightarrow \infty$. A paper investigating these questions and similar ones is under preparation.

\section{REFERENCES}

1. G. H. Hardy, Divergent series, Clarendon Press, Oxford, 1949.

2. A. Tauber, Ein Satz aus der Theorie der unendlichen Reihen, Monatsh. Math. Phys. 8 (1897), 273-277.

Technische Hochschule Stuttgart, West Germany 